\title{
O fazer do profissional de Educação Física no Centro de Atenção Psicossocial: uma análise do cuidado a partir da Política Nacional de Humanização
}

\author{
The practice of Physical Education professionals in the Psychosocial Care Center: an analysis \\ of care based on the National Humanization Policy
}

La práctica de los profesionales de Educación Física en el Centro de Atención Psicosocial: un análisis de la atención basado en la Política Nacional de Humanización

Ameliane da Conceição Reubens-Leonidio ${ }^{a, b, c, *}$ (ID), Talita Grazielle Pires de Carvalho ${ }^{a}$ (D), Ana Raquel Mendes dos Santos ${ }^{\mathrm{a}, \mathrm{d}}$

Palavras-chave

Educação física; Saúde mental; Humanização; Sistema Único de Saúde.

\section{Keywords}

Physical education; Mental health; Humanization; Health Unic System.

Palabras clave Educación física; Salud mental; Humanización; Sistema único de Salud.

\begin{abstract}
RESUMO
Estudo descritivo que teve como objetivo identificar como se apresentam valores da Política Nacional de Humanização (PNH) no fazer de profissionais de Educação Física (PEF) em Centros de Atenção Psicossocial (CAPS). Foram realizadas 4 entrevistas e 32 observações. As categorias analisadas foram autonomia do usuário; vínculo profissional-usuário; e participação coletiva na gestão. Os resultados apontaram que valores da PNH transitam no fazer do PEF em CAPS, no entanto ainda é possível identificar atitudes hospitalocêntricas. Neste sentido, reforça-se a necessidade de educação permanente; diálogo e práticas interprofissionais; e parcerias institucionais no território, como elementos importantes na produção do cuidado antimanicomial.
\end{abstract}

\begin{abstract}
This descriptive study aimed to identify how values of the National Humanization Policy (PNH) are presented in the work of Physical Education professionals (PEF) in Psychosocial Care Centers (CAPS). 4 Interviews and 32 observations were carried out. The categories analyzed were user autonomy; professional-user bonds; and collective participation in management. The results showed that values of the PNH transit in making the PEF in CAPS, however it is still possible to identify hospital-centered attitudes. In this sense, the need for permanent education is reinforced; interprofessional dialogue and practices; and institutional partnerships in the territory, as important elements in the production of anti-asylum care.
\end{abstract}

\begin{abstract}
RESUMEN
Estudio descriptivo identificado cómo se presentan los valores de la Política Nacional de Humanización (PNH) en el trabajo de los profesionales de Educación Física (PEF) en los Centros de Atención Psicosocial (CAPS). Se realizaron 4 entrevistas y 32 observaciones. Las categorías analizadas fueron la autonomía del usuario; bonos de usuarios profesionales; y participación colectiva en la gestión. Los resultados mostraron que los valores del tránsito de la PNH al hacer el PEF en CAPS, todavía es posible identificar actitudes centradas en el hospital. En este sentido, se refuerza la necesidad de una educación permanente; diálogo y prácticas interprofesionales; y asociaciones institucionales en el territorio, como elementos importantes en la producción de la atención contra el asilo.
\end{abstract}

\footnotetext{
aUniversidade de Pernambuco, Programa Associado de Pós-graduação em Educação Física UPE/UFPB. Recife, PE, Brasil. 'Instituto Federal de Pernambuco. Cabo de Santo Agostinho, PE, Brasil.

'Secretaria Municipal de Saúde do Recife, Programa Academia da Cidade. Recife, PE, Brasil.

dUniversidade de Pernambuco, Escola Superior de Educação Física. Recife, PE, Brasil.
}

\footnotetext{
*Autor correspondente:

Ameliane da Conceição Reubens-Leonidio

E-mail: ameliane.doutorado@gmail.com
} 


\section{INTRODUÇÃO}

Na micropolítica do processo de trabalho em saúde ocorrem encontros através dos quais se constroem atos de cuidado (Merhy, 2013). Para Pinheiro e Mattos (2004), o cuidado é caracterizado pela atenção, zelo e desvelo entre os sujeitos e é sustentado por práticas que permitem o diálogo e a corresponsabilidade. Insere-se, portanto, no fazer ético, político e estético da atenção à saúde mental, podendo contribuir para a constituição de um ethos antimanicomial.

Almeida e Merhy (2020) consideram que o trabalho antimanicomial em ato, enquanto ética, independe de um ideal a ser alcançado, mas é disparado através de encontros que podem gerar transformação. Nessa perspectiva, Carvalho et al. (2008) afirmam que o cuidado é uma atitude que implica numa disponibilidade do profissional para ser afetado, seja pelos sentidos, afetos ou abertura ao outro. Do ponto de vista estético, o cuidado pressupõe um processo criativo de promoção da vida, considerando a abertura para diversos modos de existência (Cavallini, 2020), e revela-se, também, no direito à cidadania, democracia, reordenamento de poderes e emancipação, como atitude política (Ballarin et al., 2010).

De tal forma, o cuidado é o produto do trabalho em saúde (Merhy, 2007), que requer a utilização da caixa de ferramentas de cada profissional. Para Amarante e Torre (2017), o cuidado antimanicomial faz parte da dimensão técnico-assistencial da reforma psiquiátrica e para sua efetivação, é preciso que as ferramentas de cada trabalhador sejam compartilhadas a partir da interdisciplinaridade, como trabalho de campo; ou utilizadas de modo singular nas práticas de núcleo, mas sem perder de vista os princípios da reforma psiquiátrica.

No Centro de Atenção Psicossocial (CAPS) o processo de trabalho ocorre por meio da composição de equipes multiprofissionais, nas quais podem fazer parte profissionais de Educação Física (PEF) (Brasil, 2004). Estudos atuais tratam das experiências de PEF em CAPS, sobretudo a partir das atividades de núcleo (Furtado et al., 2014; Silva et al., 2017; Daltio et al., 2020; Figueiredo et al., 2020). No entanto, é notório que a carência da temática saúde mental na formação ainda é um dos principais entraves da atuação, o que é preocupante visto que mudanças no lócus de tratamento em saúde mental não determinam a superação de práticas hospitalocêntricas (Liberato; Dimenstein, 2013). Assim, é imprescindível para PEF que já se encontram nos serviços de saúde mental, além do trabalho interprofissional, recursos como educação permanente e avaliações do próprio fazer, considerando que este precisa dialogar constantemente com uma função terapêutica (Wachs, 2017).

No cadernos humanizaSUS n.5, que trata a Política Nacional de Humanização (PNH) como transversal à Política Nacional de Saúde Mental, percebem-se modos de fazer no trabalho em saúde que podem contribuir para o reforço da atenção humanizada na saúde mental (Brasil, 2015). No mesmo documento, Yasuí (2015) afirma que tais políticas partilham dos "mesmos princípios e posicionamentos ético-estético e político" (p.19), e elenca dentre outros aspectos, o fomento da autonomia dos usuários, o estabelecimento de vínculo e participação coletiva na gestão, como força de resistência à hegemonia de práticas hospitalocêntricas ainda bastante presentes na atenção à saúde mental. Neste sentido, a presente pesquisa buscou identificar como se apresentam aspectos da PNH no fazer dos PEF em CAPS, a fim de provocar reflexões para avanços no cuidado antimanicomial.

\section{PERCURSO METODOLÓGICO}

Trata-se de um estudo descritivo de campo, de abordagem qualitativa, que se constituiu de um recorte da pesquisa intitulada "Saúde Mental, prática corporal e emoção dos usuários dos Centros de Atenção Psicossocial do Estado de Pernambuco: uma análise a partir da pluralidade metodológica". Esse desenho foi adotado pela necessidade de aproximação do pesquisador com as experiências do cenário pesquisado, para a compreensão da realidade social e descoberta de novos problemas (Flick, 2009; Minayo, 2010).

Além disso, a pesquisa qualitativa é uma abordagem metodológica condizente com a necessidade de análise em profundidade na atenção psicossocial e possui uma "[...] aproximação com o paradigma emergente ao qual se afilia o próprio processo de Reforma Psiquiátrica brasileira" (Ramos et al., 2019, p. 840).

O estudo foi realizado em 4 CAPS do município de Recife. Para a triangulação dos dados, como sugere Flick (2009), foram utilizados: 32 horas de observações não-participante do grupo da Educação Física, tendo como instrumento um roteiro contendo aspectos de participação, emoções e interações sociais; e entrevistas semiestruturadas individuais com 4 PEF dos serviços, as quais foram gravadas e permitiram, como sugere Banks (2009), a liberdade de introduzir novas perguntas e fazer intervenções durante o processo para o aprofundamento do nível das informações e opiniões. Esses instrumentos foram testados num estudo piloto, realizado num CAPS de um município vizinho (Cabo de Santo Agostinho), e submetidos à apreciação de cinco professores de diferentes Instituições de Ensino Superior (IES) brasileiras.

Os dados das observações foram digitados e catalogados, enquanto os dados das entrevistas foram transcritos e tratados à luz da Análise de Conteúdo de Bardin (2011), que tem como objetivo descobrir as relações existentes entre o conteúdo das falas e os aspectos exteriores (Santos, 2012). O tema foi tomado como uma assertiva sobre os assuntos, para o qual foram considerados uma simples sentença ou o conjunto de sentenças ou um parágrafo, como sugerido por Franco (2003). Foi possível, então, identificar os núcleos de sentido que nortearam as categorias. 
Como instrumento para organização dos dados, foi utilizado o software Atlas ti. versão 7.0, através do qual seguiu-se as etapas propostas por Bardin (2011): leitura flutuante; ordenação dos dados; releitura e codificação do material, utilizando a sigla PEF e o número para cada profissional entrevistado; organização das categorias e análise final do material. As categorias deram-se $a$ priori, a partir da articulação PNH e a Política Nacional de Saúde Mental: 1. autonomia dos usuários; 2. vínculo profissional-usuário; e 3. participação na gestão. 0 software GoDiagram Express versão 2.6.2 foi utilizado para a construção da figura ilustrativa.

Essa pesquisa foi autorizada pela Prefeitura da Cidade do Recife e aprovada pelo Comitê de Ética em Pesquisa com seres humanos da Universidade de Pernambuco, sob o CAAE 23424613.4.0000.5207. Foram observadas as diretrizes estabelecidas na Resolução $466 / 12$, do Conselho Nacional de Saúde, e todos os participantes assinaram o Termo de Consentimento Livre e Esclarecido, permitindo a coleta e a utilização dos dados, sendo-lhes assegurados todos os direitos.

\section{CENÁRIO DO ESTUDO}

Entre os serviços da Rede de Atenção Psicossocial (RAPS) de Recife estão 8 CAPS destinados ao cuidado de pessoas acima de 15 anos em sofrimento psíquico. No momento da pesquisa, 4 PEF do Programa Academia da Cidade (PAC) atuavam nesses espaços com o objetivo de promover a integração social e familiar, através de atividades terapêuticas e projetos individuais, visando a autonomia e o exercício de cidadania (Cruz et al., 2013).
Tendo que dividir a carga horária entre o polo do PAC e CAPS, PEF tinham como atribuições de campo a participação na reunião clínico-institucional, a realização de intervenções interdisciplinares e apenas um entrevistado relatou desempenhar a função de Técnico de Referência (TR), profissional responsável pelo acompanhamento do Projeto Terapêutico Singular (PTS) do usuário. Existiam posicionamentos contrários à disposição do trabalho: "[...] eu acho que a gente teria que ser ou academia da cidade ou CAPS. Eu acho que é ponto negativo com relação a isso" (PEF_02). Tal perspectiva não era unânime, porém o cenário implicava em fragilidades no fazer de campo, evidenciando desigualdades entre PEF e os outros técnicos das equipes.

Como atribuição de núcleo, todos eram responsáveis pelo Grupo Movimento (GM), no qual ocorriam práticas corporais, práticas integrativas, jogos de salão e atividades de educação em saúde, como mostra a Figura 1.

Os entrevistados revelaram uma dificuldade inicial em organizar as práticas sob o ponto de vista terapêutico, visto que não tinham formação para a atuação em saúde mental e desconheciam os princípios da reforma psiquiátrica. Em função disso, sugeriam momentos de educação permanente que pudessem fundamentar o fazer de núcleo, mas ainda eram escassos. Assim, a caixa de ferramentas dos PEF nos CAPS para atuação no núcleo era composta essencialmente pela transposição das experiências prévias de outros locais de atuação, como escolas, clubes, clínicas e do próprio PAC, que constituem suas tecnologias leve-duras, além de tecnologias leves como vínculo e tecnologias duras como os próprios instrumentos materiais, segundo a conceituação de Merhy (2007).

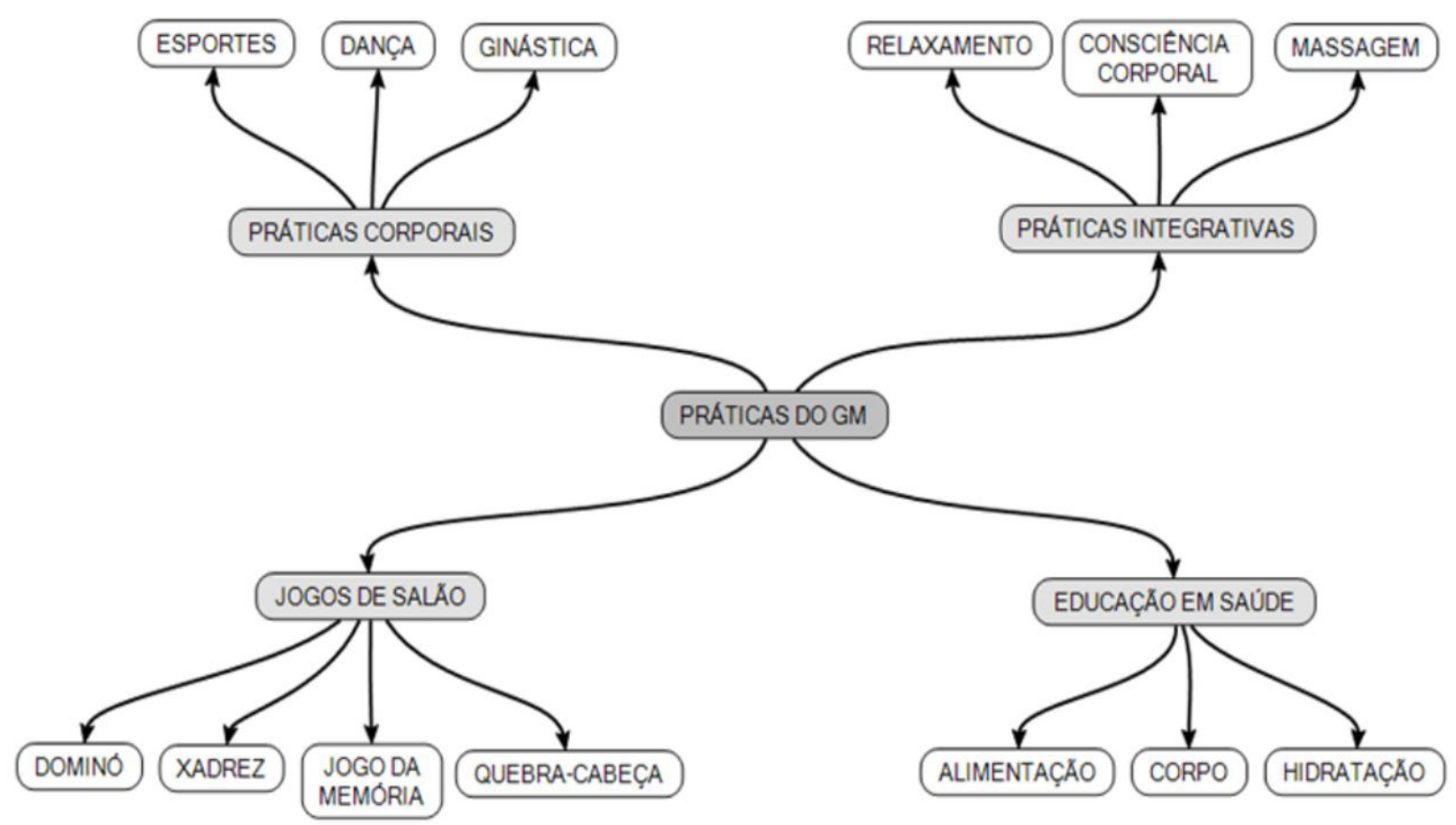

Figura 1. Práticas do GM. Fonte: os autores. 
Diante do cenário apresentado e tomando como base o preceito de que é preciso uma constante observação das práticas de cuidado em saúde mental, a fim de superar propostas manicomiais e em observância também à articulação com a $\mathrm{PNH}$, serão analisadas três categorias: 1 . autonomia dos usuários; 2 . vínculo profissional-usuário; e 3. participação coletiva na gestão do fazer dos PEF nos CAPS considerando as práticas do núcleo profissional.

\section{AUTONOMIA DOS USUÁRIOS: ENTRE A RESPONSABILIZAÇÃO E A CORRESPON- SABILIZAÇÃO}

Embora existam diferentes interpretações para o conceito de autonomia, pretende-se aqui considerá-la como a possibilidade do sujeito gerir sua vida, realizando suas escolhas e estabelecendo redes sociais para transitar no território. Na PNH, a autonomia é colocada como a possibilidade do exercício do papel de cidadão, a partir do conhecimento e efetivação de direitos e responsabilidades (Brasil, 2010). Espera-se, desta forma, que os usuários do SUS possam gerir e cuidar de sua saúde como sujeitos, superando a ideia de paciente sob o qual são realizadas prescrições impositivas.

Na saúde mental, os CAPS têm o papel estratégico de articulação da RAPS e promoção da autonomia dos usuários, por meio de um processo de corresponsabilização (Brasil, 2013). Assim, como resposta da atenção direta, do fazer que se apresenta no trabalho em ato, a autonomia pode ser percebida através do autocuidado e valorização da própria vida. O documento sobre CAPS e Unidades de Acolhimento (UA), publicado em 2013 pelo Ministério da Saúde (MS), considera as práticas corporais como ferramentas que podem ajudar a fortalecer esses elementos, quando cita a possibilidade de melhorar a “[...] percepção corporal, a autoimagem, a coordenação psicomotora, compreendidos como fundamentais ao processo de construção de autonomia, promoção e prevenção em saúde" (Brasil, 2013, p. 9).

No presente estudo, os entrevistados relataram modificações dos usuários, no que se refere à autonomia após a inserção no GM, ainda que essas modificações fossem discretas, como apresentado no relato a seguir: "[...] às vezes faz atividade em equilíbrio e só por eles conseguirem ficar um tempo maior equilibrados, então isso já é o máximo pra eles [...] [aumentam as] possibilidades de autonomia deles, de buscar essa autonomia em pequenas coisas [...]" (PEF_03). Esse exemplo demonstra uma superação dos próprios limites, a não desistência em realizar um movimento corporal e um olhar para si enquanto sujeito capaz.

Para Luz (2012), tentar superar limites representa uma atitude de vitalidade e pode implicar em autonomia, mas é preciso atentar para que essa atitude não aumente os riscos de danos à saúde. Era nesse contexto que a educação em saúde ganhava um especial destaque, por propiciar momentos para trocas de saberes a partir do diálogo. Através dos registros nos roteiros de observação, identificou-se que, por vezes, os usuários mantinham atitudes, com relação à práticas corporais, que poderiam ser danosas à saúde, especialmente quando não estavam nos CAPS. Em resposta, foi possível notar duas tendências nas entrevistas dos PEF: uma que evocava a responsabilização e outra a corresponsabilização.

Na primeira tendência transitava um discurso de responsabilização do usuário pela adoção de um comportamento de risco à saúde. Esta abordagem está ligada à homogeneização do estilo de vida, vigilância e controle de comportamentos. Foram registradas falas como: "[...] eles tem preguiça, né? De sair de casa pra fazer, [...] distúrbio mental faz com que eles tenham essa preguiça [...]" (PEF_04).

Firmo e Jorge (2015) consideram que há referenciais que se equivocam ao traduzir autonomia como responsabilização dos sujeitos pelo próprio adoecimento, pois dessa forma haveria uma capacidade das pessoas agirem sobre os determinantes independente do contexto. Neste sentido, para Dutra et al. (2017), a autonomia nem sempre deve corresponder a uma independência absoluta, mas pode ocorrer de forma mediada com suporte dos profissionais.

Na segunda tendência, foi possível evidenciar um processo educativo, de modo que os usuários pudessem pensar sobre a prática não como repressão a um comportamento de risco, mas como meio reflexivo. Essa tendência fortalece ainda mais a autonomia dos usuários, pois para Jorge et al. (2011), está relacionada à ampliação da capacidade de compreender e atuar sobre si, sendo o grau de autonomia na saúde mental medido pelo autocuidado, compreensão do processo saúde-doença, uso do poder e compromisso contratual. Assim, apresenta-se como uma possibilidade dos usuários buscarem os PEF como apoio, o que implica na construção de corresponsabilização.

No contexto do estudo, já considerando as fragilidades apontadas no fazer de campo, os PEF tinham pouca afinidade com as ferramentas utilizadas nos CAPS, como acolhimento, PTS e a função de TR, mas muitos demonstravam sensibilidade para a escuta qualificada, o que favorecia a construção do vínculo profissionalusuário, que será abordado mais adiante.

\section{VÍNCULO PROFISSIONAL-USUÁRIO: IMPLICAÇÕES NO INTRA E NO EXTRA MUROS}

Vínculo implica em envolvimento, em encontros de sujeitos e subjetividades, é um recurso terapêutico que se dá pela corresponsabilização, através do fortalecimento das relações no serviço, que pode também servir de apoio para aproximações com o território. Para Barbosa e Bosi (2017), na Saúde Coletiva vínculo pode ser considerado um dispositivo ou uma ferramenta relacional. $O$ vínculo na referência à caixa de ferramentas por Seixas et al. (2019) é uma tecnologia leve, que se apresenta no Trabalho Vivo em ato. No caso deste estudo, o trabalho 
antimanicomial em ato como sugerem Almeida e Merhy (2020).

Na pesquisa realizada por Leonidio et al. (2013), também em Recife, o vínculo era a ferramenta que se estabelecia de forma mais evidente no processo de trabalho do PEF nos CAPS. No estudo atual, percebe-se que o vínculo ainda se apresenta importante, inclusive pelas possibilidades ampliadas de expressões a partir das práticas corporais, como se apresenta no trecho a seguir: "[...] eu já tive usuário aqui que nunca falou nada, [...] e falava com o corpo muita coisa, e a partir disso a gente foi conseguindo fazer um resgate da história dele [...]" (PEF_03).

O vínculo permite o estabelecimento de confiança na relação entre profissional-usuário, favorecendo trocas e diálogo. Muitos PEF, independente do momento de realização do $G M$, mantinham uma atitude de circular nos espaços dos CAPS, o que chamavam de corpo-a-corpo, favorecendo assim a aproximação e o diálogo com os usuários. Nas observações, foi possível identificar situações em que os usuários relatavam fatos importantes de suas vidas para PEF, muitas vezes suprimindo a informação dos próprios TR.

Por outro lado, vínculo PEF-usuário ainda era incipiente para a adesão/manutenção em programas/ projetos com práticas corporais no território, sobretudo no PAC, como revela o seguinte depoimento: "[...] são poucos, de virem falar comigo e dizer: professor, [...] comecei a participar das aulas lá [...], já teve até usuário aqui que já fez aula comigo lá, são poucos, não são muitos não". (PEF_04). Essa aproximação PEF-usuário era, também, uma proposta adotada pela gestão municipal como estratégia de reinserção social, através do PAC ou de outros espaços que tivessem as práticas corporais como ferramenta de cuidado, mas não se mostrou efetiva.

Os PEF acreditavam que deveria haver pactuações entre a gestão municipal e outros espaços na estratégia de reinserção, como demonstra o trecho a seguir: "[...] então se houvesse um vínculo da prefeitura com essas entidades próximas aos CAPS [...], facilitaria bastante [...] essa questão das parcerias [...]" (PEF_03). Na mesma linha, Furtado et al. (2017) sugere uma desinstitucionalização das práticas dos CAPS e uma institucionalização das parcerias intersetoriais para a utilização de outros espaços no território. Nota-se, portanto. que o vínculo pode favorecer o trabalho interno, o fazer do PEF intra CAPS, mas sem parcerias institucionais, pouco repercute nas possibilidades extra CAPS.

\section{PARTICIPAÇÃO COLETIVA NA GESTÃO: VERTICALIDADE, HORIZONTALIDADE E TRANSVERSALIDADE NA MICROPOLÍTICA DO GRUPO MOVIMENTO}

Nos CAPS são utilizadas estratégias de participação coletiva de usuários e familiares na gestão, a fim garantir a participação democrática no cuidado. Como exemplo, têm-se a participação de usuários e familiares em conferências de saúde, conselho gestor das unidades de saúde e assembleias (Campos et al., 2009). Durante as observações em um dos CAPS, pôde-se acompanhar uma assembleia que ocorreu no horário do GM. O momento foi registrado e despertou a atenção especialmente pela solicitação dos usuários para inclusão de jogos e atividades de lazer na rotina do CAPS, o que revelava um interesse pela ampliação das práticas que já eram realizadas no GM.

Da mesma forma, nas práticas de núcleo profissional é extremamente importante a participação do usuário, tendo em vista as possibilidades de utilização de ferramentas que repercutirão efetivamente no seu cuidado. No GM, por sua vez, o planejamento, condução e avaliação da práticas ocorriam a partir de três modelos: vertical, horizontal e transversal.

O primeiro modelo identificado foi o verticalizado, no qual a organização se dava através do planejamento, condução e avaliação individual, realizada pelo próprio $P E F$, especialmente quando não se percebia o desejo dos usuários em colaborar: "normalmente a gente não tem essa resposta, ficam calados e o que eu trouxer tá bom. Normalmente o que eles fazem é isso" (PEF_04). Percebe-se na fala do PEF ainda uma atitude de responsabilização, sobretudo do ponto de vista político do cuidado.

Para Penido e Romagnoli (2018), a verticalização das ações no cuidado provocam uma introjeção de normas e demandas institucionais, repercutindo em sujeição. Desta forma, mantém-se atitudes manicomiais, pois desvincula-se da noção de autonomia e protagonismo dos usuários. O usuário, neste modelo, é percebido através do estigma social, que Goffman (1984) coloca como uma condição para olhar o sujeito enquanto inoperante socialmente. Portanto, é necessário avaliar as questões que mantém os usuários distantes da gestão do seu cuidado, considerando como ocorrem as relações de poder e os vínculo entre profissional-usuários.

No segundo modelo, horizontalizado, ocorria a participação coletiva na gestão do grupo em parceria com os usuários: "[...] a gente escolhe junto com eles, a gente senta de três em três meses e planeja o que vai ser feito nesses três meses seguintes" (PEF_02). Tal proposta dava-se de forma dialogada e em alguns momentos recorria-se à votação para elencar as práticas, mas sempre numa perspectiva de pensar a construção do GM coletivamente para que essa atitude pudesse transpor o próprio espaço, como revela-se na fala de um PEF: "[...] a questão de trabalhar junto com o outro, que isso leva pra vida, né? [...]" (PEF_01).

A horizontalidade no cuidado se apresenta de forma diferenciada da noção de verticalidade por permitir a participação do usuário nas decisões do grupo. No entanto, Penido e Romagnoli (2018) ainda consideram a horizontalidade distante da noção de autonomia, pois 
baseados nas ideias de Guattari (1987), essaé uma condição na qual cria-se uma coesão grupal, um nivelamento diante da situação, que a grosso modo ainda não permite a produção do sujeito. O filósofo propõe a ideia de transversalidade em contrapartida aos dois modelos apresentados, considerando os afetos e quando busca-se por um grupo sujeito, produtor de novas realidades e invenções. A transversalidade acontece na comunicação máxima entre diferentes níveis e sentidos (Guattari, 1987).

Foi possível observar um movimento aproximado da noção de transversalidade quando os usuários, além da participação no planejamento, atuavam como autores de oficinas ou exerciam o papel de protagonista na tomada de decisões envolvendo práticas corporais, produzindo, assim autonomia. Neste sentido, Wachs (2008), citando com exemplo o jogo de futebol, considera que nesse pequeno universo, os usuários podem se tornar protagonistas de suas próprias vidas.

Os arranjos apresentados na participação coletiva na gestão do GM demonstram como no trabalho em ato podem ser estabelecidas diferentes relações de poder que implicam na dimensão política do cuidado. Essas diversidades não são exclusivas da Educação Física, mas como colocam Penido e Romagnoli (2018), são coexistentes nas práticas de saúde, o que sugere tensionamentos constantes e a necessidade de análise e políticas para redução de hierarquias e valorização de práticas que dão vida aos sujeitos individuais e coletivos.

\section{CONSIDERAÇÕES FINAIS}

O cuidado ético-estético-político na saúde mental pode ser posto em prática a partir do reforço de valores para humanização das práticas de saúde através da $\mathrm{PNH}$, que torna-se importante na medida em que nos convoca a olhar para a prática profissional, sempre na perspectiva das singularidades dos sujeitos. De tal modo, ao analisar a autonomia do usuário, vínculo profissional-usuário e participação coletiva na gestão diante das práticas de PEF em CAPS, pôde-se perceber atitudes que ainda refletem o modelo hegemônico, no qual a doença ou mesmo o estigma da impotência, aparecem anulando o sujeito.

Esse cenário reforça a necessidade de ampliação da educação permanente e práticas interprofissionais, como elementos importantes na constituição do PEF enquanto profissional da saúde mental. No entanto, embora tenham sido apontadas dificuldades iniciais, especialmente em função da carência da temática saúde mental na formação inicial, coexistiam potências no fazer do PEF nos CAPS: atitudes de corresponsabilização que educam numa perspectiva de valorização da vida, vínculos fortalecidos intra CAPS e reflexões para ampliação das possibilidades de contribuição na reinserção social, transversalidades na participação coletiva do GM, além de reflexões sobre parcerias institucionais. Nesta perspectiva, acreditamos que seja um caminho para um fazer antimanicomial.

Vale salientar que após a pesquisa, os PEF decidiram suspender as atividades nos CAPS de Recife, como uma tentativa de tensionar a gestão para vinculação ao serviço e ampliação dos recursos para educação permanente na saúde com foco na saúde mental, mas até o presente momento não obteve-se retorno das solicitações.

\section{FINANCIAMENTO}

A primeira autora recebeu apoio financeiro da Fundação de Amparo à Ciência e Tecnologia do Estado de Pernambuco (FACEPE).

\section{CONFLITOS DE INTERESSE}

Os autores declaram não ter conflitos de interesse.

\section{AGRADECIMENTOS}

Agradecemos à Prof. Dra. Clara Maria Silvestre Monteiro de Freitas e aos profissionais de Educação Física que se dispuseram a participar da pesquisa.

\section{REFERÊNCIAS}

Almeida SA, Merhy EE. Micropolítica do trabalho vivo em saúde mental: composição por uma ética antimanicomial em ato. Rev Psicol Polit. 2020;20(47):65-75.

Amarante P, Torre EHG. Loucura e diversidade cultural: inovação e ruptura nas experiências de arte e cultura da Reforma Psiquiátrica e do campo da Saúde Mental no Brasil. Interface. 2017;21(63):763-74. http://dx.doi.org/10.1590/180757622016.0881.

Ballarin MLGS, Carvalho FB, Ferigato SH. Os diferentes sentidos do cuidado: considerações sobre a atenção em saúde mental. O. Mundo Saude. 2010;34(4):444-50. http://dx.doi. org/10.15343/0104-7809.20104444450.

Banks M. Dados visuais para pesquisa qualitativa. Tradução J. Fonseca. Porto Alegre: Penso; 2009.

Barbosa MIS, Bosi MLM. Vínculo: um conceito problemático no campo da Saúde Coletiva. Physis Rev Saúde Coletiva. 2017;27(4):1003-22. http://dx.doi.org/10.1590/s010373312017000400008.

Bardin L. Análise de conteúdo. Lisboa, Portugal: Editora 70; 2011.

Brasil. Ministério da Saúde. Saúde Mental no SUS: os Centros de Atenção Psicossocial [Internet]. Brasília; 2004 [citado 2020 Ago 1]. Disponível em: https://www.saude.sc.gov.br/index. php/documentos/atencao-basica/saude-mental/manual-decaps/2874-manual-de-caps/file

Brasil. Política Nacional de Humanização [Internet]. Brasília: HumanizaSUS; 2010 [citado 2020 Ago 1]. Disponível em: http://bvsms.saude.gov.br/bvs/publicacoes/politica_ nacional_humanizacao_pnh_folheto.pdf

Brasil. Ministério da Saúde. Manual de estrutura física dos Centros de Atenção Psicossocial e Unidades de Acolhimento: orientações para elaboração de projetos de construção, reforma e ampliação de CAPS e de UA como lugares da atenção psicossocial nos territórios [Internet]. Brasília; 2013 [citado 2020 Ago 1]. Disponível em: http://189.28.128.100/ dab/docs/sistemas/sismob/manual_ambientes_caps_ua.pdf

Brasil. Ministério da Saúde. Secretaria de Atenção à Saúde. Política Nacional de Humanização [Internet]. Brasília: HumanizaSUS; 
2015. (Caderno HumanizaSUS; 5: Saúde mental) [citado 2020 Ago 1]. Disponível em: http://bvsms.saude.gov.br/bvs/ publicacoes/saude_mental_volume_5.pdf

Campos GWS. Saúde pública e saúde coletiva: campo e núcleo de saberes e práticas. Cien Saude Colet. 2000;5(2):219-30. http://dx.doi.org/10.1590/S1413-81232000000200002.

Campos RTO, Furtado JP, Passos E, Ferrer AL, Miranda L, Gama CAP. Avaliação da rede de centros de atenção psicossocial: entre a saúde coletiva e a saúde mental. Rev Saude Publica. 2009;43(1, Supl 1):16-22. http://dx.doi.org/10.1590/S003489102009000800004.

Carvalho LB, Bosi MLM, Freire JC. Dimensão ética do cuidado em saúde mental na rede pública de serviços. Rev Saude Publica. 2008;42(4):700-6. http://dx.doi.org/10.1590/S003489102008000400017. PMid:18553040.

Cavallini FM. CAPS, ateliês e oficinas: artes no mundo, mundos na arte. Fractal Rev Psicol. 2020;32(1):40-5.

Cruz D, Albuquerque G, Damascena W. Programa Academia da Cidade do Recife: minimizando contrastes sociais. 2013 [citado 2020 Ago 1]. Disponível em: http://dssbr.org/site/ experiencias/programa-academia-da-cidade-do-recifeminimizando-contrastes-sociais/

Daltio GL, Abib LT, Gomes IM. Possibilidades e tensões no trabalho com as práticas corporais no cuidado em saúde mental: reflexões construídas em um CAPS II na cidade de Serra/ES. Pensar Prát. 2020;23:e56692. http://dx.doi.org/10.5216/ rpp.v23.56692.

Dutra VFD, Bossato HR, Oliveira RMP. Mediar a autonomia: um cuidado essencial em saúde mental. Esc Anna Nery. 2017;21(3):1-8.

Figueiredo SMT, Oliveira BN, Espírito-Santo G. Atuação do profissional de educação física em CAPS representada pelos demais profissionais do serviço. Pensar Prát. 2020;23:e56378. http://dx.doi.org/10.5216/rpp.v23.56378.

Firmo AAM, Jorge MSB. Experiências dos cuidadores de pessoas com adoecimento psíquico em face à reforma psiquiátrica: produção do cuidado, autonomia, empoderamento e resolubilidade. Saude Soc. 2015;24(1):217-31. http://dx.doi. org/10.1590/S0104-12902015000100017.

Flick U. Uma introdução à pesquisa qualitativa. 3. ed. Porto Alegre: Artmed; 2009.

Franco MLPB. Análise de conteúdo. Brasília: Plano; 2003.

Furtado RP, Oliveira MFM, Sousa MF, Vieira PS, Neves RLR, Rios $\mathrm{GB}$, et al. O trabalho do professor de educação física no Caps: aproximações iniciais. Movimento. 2014;21(1):41-52. http:// dx.doi.org/10.22456/1982-8918.43457.

Furtado RP, Sousa MF, Martinez JFN, Rabelo NS, Oliveira NSR, Simon WJ. Desinstitucionalizar o cuidado e institucionalizar parcerias: desafios dos profissionais de Educação Física dos CAPS de Goiânia em intervenções no território. Saude Soc. 2017;26(1):183-95. http://dx.doi.org/10.1590/s010412902017169101.

Goffman E. Estigma: notas sobre a manipulação da identidade deteriorada. Rio de Janeiro: Zahar Editores; 1984.

Guattari F. Revolução molecular: pulsações políticas do desejo. São Paulo: Brasiliense; 1987.

Jorge MSB, Pinto DM, Quinderé PHD, Pinto AG, Sousa FSP, Cavalcante CM. Promoção da Saúde Mental: tecnologias do Cuidado: vínculo, acolhimento, co-responsabilização e autonomia. Cien Saude Colet. 2011;16(7):3051-60. http:// dx.doi.org/10.1590/S1413-81232011000800005.

Leonidio ACR, Lemos EC, Silva PPC, Freitas CMSM. O profissional de Educação Física no Centro de Atenção Psicossocial: percepção dos limites e potencialidades no processo de trabalho. Pesquisas e Práticas Psicossociais. 2013;8(2):157-65.

Liberato MTC, Dimenstein M. Arte, loucura e cidade: a invenção de novos possíveis. Psicol Soc. 2013;25(2):272-81. http://dx.doi. org/10.1590/S0102-71822013000200004.

Luz MT. Novos saberes e práticas em saúde coletiva: Estudos sobre racionalidades médicas e atividades corporais. 3. ed. São Paulo: Hucitec; 2012.

Merhy EE. Os CAPS e seus trabalhadores: no olho do furacão antimanicomial. Alegria e alívio como dispositivos analisadores. In Mehry EE, Franco TB, editores. Trabalho, produção de cuidado e subjetividade: textos reunidos. São Paulo: Hucitec; 2013. p. 213-25.

Merhy EE. Saúde: a cartografia do trabalho vivo. 3. ed. São Paulo: Editora Hucitec; 2007.

Minayo MCS. O desafio do conhecimento: pesquisa qualitativa em saúde. 12. ed. São Paulo: Hucitec; 2010.

Penido CMF, Romagnoli RC. Apontamentos sobre a clínica da autonomia na promoção da saúde. Psicol Soc. 2018;30(0):e173615. http://dx.doi.org/10.1590/18070310/2018v30173615.

Pinheiro R, Mattos RA, editores. Cuidado: as fronteiras da integralidade. São Paulo-Rio de Janeiro: Hucitec-Abrasco; 2004.

Ramos DKR, Paiva IKS, Guimaraes J. Pesquisa qualitativa no contexto da Reforma Psiquiátrica brasileira: vozes, lugares, saberes/fazeres. Cien Saude Colet. 2019;24(3):839-52. http:// dx.doi.org/10.1590/1413-81232018243.00512017.

Santos FM. Análise de conteúdo: a visão de Laurence Bardin. Rev Educ. 2012;6(1):383-7.

Seixas CT, Baduy RS, Cruz KT, Bortoletto MSS, Slomp H Jor, Merhy EE. O vínculo como potência para a produção do cuidado em Saúde: o que usuários-guia nos ensinam. Interface. 2019;23:e170627. http://dx.doi.org/10.1590/ interface.170627.

Silva TMC, Santos FM, Silva RCB, Oliveira HLR, Ilha PV, Graup S. Educação Física e Saúde Mental: atuação profissional nos Centros de Atenção Psicossocial. Pensar Prát. 2017;20(3):53951.

Wachs F. Educação Física e o cuidado em saúde. Saúde em Redes. 2017;3(4):339-49. http://dx.doi.org/10.18310/24464813.2017v3n4p339-349.

Wachs F. Educação Física e saúde mental: uma prática de cuidado emergente em Centros de Atenção Psicossocial (CAPS) [dissertação]. Porto Alegre: Universidade Federal do Rio Grande do Sul; 2008.

Yasuí S. Entre o cárcere e a liberdade: apostas na produção cotidiana de modos diferentes de cuidar. In: Brasil. Ministério da Saúde. Secretaria de Atenção à Saúde. Política Nacional de Humanização. Brasília: HumanizaSUS; 2015. (Caderno HumanizaSUS; 5: Saúde mental) [citado 2020 Ago 1]. Disponível em: http://bvsms.saude.gov.br/bvs/publicacoes/saude_ mental_volume_5.pdf 